\title{
Divergent Perspectives on Pediatric Communication Disorders in Extremely Preterm Birth: A Case Study and Considerations for Evidence Based Practice
}

\author{
Melissa Randazzo \\ Department of Communication Sciences \& Disorders, Adelphi University, New York, United States
}

\begin{abstract}
This case report addresses the linguistic and motor speech development of a child born prematurely at 24 weeks' gestation. Because of the complex nature of this child's developmental trajectory he was evaluated and treated for suspected Childhood Apraxia of Speech, a disorder which is difficult to differentially diagnose. Now at age 4 years his clinical presentation is consistent with expressive and receptive language disorder with mild dysarthria. This work presents for consideration the inherent difficulty in diagnosing and treating children with rare and underreported conditions such as extremely preterm birth and very low birthweight. Additionally, this report explores how clinicians with distinct theoretical orientations (e.g., motor vs. linguistic) viewed this child from divergent perspectives, causing clinical disagreement. Consideration is invited of how we view communication disorders in complex developmental situations, informed by both historical perspectives and existing research on speech and language development in children born extremely preterm.
\end{abstract}

Keywords: Preterm birth, Childhood apraxia of speech, Pediatric speech sound disorders

\section{INTRODUCTION}

This case report presents the speech-language development as well as evaluation and treatment history of "Ivan" (pseudonym), a child born prematurely at 24 weeks' gestation. Due to his complex medical background and ongoing health issues, Ivan's developmental trajectory is unconventional and requires the support of various interventionists across disciplines. While Ivan presents with gross and fine motor difficulties, necessitating both physical and occupational therapy, this report focuses exclusively on the development of speech, language, and associated cognitive skills in this child. Speech and language development in premature infants has been notoriously difficult to study, as there is wide heterogeneity in the population. Children born prematurely have complex developmental trajectories, which are multifaceted and often complicated by other health issues. Examination of such cases illustrates how, depending on clinicians' conceptualizations of speech and language disorders as motoric or language-based difficulties, different aspects of development may be over- or under-emphasized.

Ivan's case is unique as he belongs to two high-risk categories of premature birth. Ivan classifies as a child born extremely preterm, born at 24 weeks' gestation. Further, he classifies as a micropreemie and very low birthweight child, weighing just 440 grams

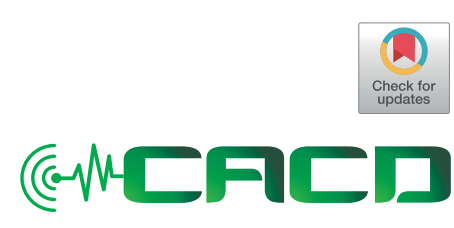

Received: January 16, 2018 Accepted: April 16, 2018

\section{Correspondence: Melissa Randazzo}

Department of Communication Sciences \& Disorders, Adelphi University, New York, United States

Tel: +516-877-4769

Fax: +516-877-4783

E-mail:mrandazzo@adelphi.edu 
at birth. Children born at or before 24 weeks' gestation are at the highest risk for death or major morbidity [1]. Survival rates of children born at 24 weeks are estimated at $66 \%$, and although still within a challenging time window, are better than in previous decades [2]. While survival rates for infants born before the threshold of viability (less than 28 weeks' gestation) are increasing, there are very few studies and reports documenting development and later outcomes of these children. Therefore, we have a limited understanding of the complex linguistic and cognitive development of these children, as well as wide gaps in the literature regarding their response to intervention and best practices. This report outlines Ivan's speech and language development through evaluation reports and treatment progress under the care of two different speech-language pathologists. Each clinician took a distinct approach in how they viewed, assessed, and treated Ivan's communication impairment, resulting in clinical disagreement. This unconventional situation, of a child with a rare and underreported condition being assessed and treated by two clinicians who hold distinct views on speech disorders, is the impetus for this case report.

\section{Speech and language development in premature children}

Speech and language development in premature children has been particularly difficult to study, due to the wide heterogeneity in the population, as well as small sample size and obstacles in controlling for social and environmental factors. In addition to baseline physical or neurological differences, speech and language development may be negatively impacted by the complex medical attention required by the situation, thus interfering with caregiver and child interactions [3]. For children classified as extremely preterm, the difficulties are greater due to the evolving nature of this population: although infants traditionally at the lower limits of viability have increasing survival rates in recent years, this also necessitates more intensive medical intervention and ongoing care, with correspondingly complex developmental sequelae.

A lag in development of communication skills is noted quite early in preterm infants. Neurophysiological differences in speech perception of native phonemes in preterm children as young as 6 months old have been identified [4]. Early turntaking vocalizations in response to child-directed speech are less frequent in premature infants compared to full-term peers [5]. There are limited detailed data regarding early motor speech production in children born prematurely. Children born preterm tend to have smaller expressive vocabularies at age two [6], thus compounding the difficulty of generating detailed reports of developmental speech patterns. Studies with small samples show that very low birthweight children have delayed speech sound development compared to full term peers before age 2 [7]. Other reports indicate that at schoolage, verbal children born extremely preterm have more speech articulation errors than their peers, even when controlling for cognitive status [8]. However, others suggest that delays in speech sound production are more apparent early on and dissipate as the child matures [9-11]. The role of chronic lung disease in this population [12] as a factor limiting speech production capabilities has also been underexplored, and could account for some of the discrepancies between studies of speech errors.

Foster-Cohen and colleagues [6] examined a cohort of children born before or at 33 weeks' gestation when they reached two years old. They found a linear relationship between gestational age and later language outcomes, particularly for vocabulary size, quality of word use, and morphological and syntactic complexity. Children born extremely preterm, before 28 weeks' gestation, presented with the greatest vocabulary impairment, with a marked difficulty understanding decontextualized language. Luoma and colleagues [13] similarly found language deficits in children born prematurely when they reached 5 years of age. When removing children with neurological disabilities from the preterm sample, children born at or before 32 weeks presented with significant deficits in speech production, receptive language, language formulation, and intelligence quotient compared to typically developing peers. This study further highlighted that these delays can be persistent and pervasive, even when controlling for individual and family factors. These differences or deficits in language development become more apparent as a child gets older. For instance, Luoma et al. showed marked differences in premature children in rapid automized naming - requiring more processing time - and understanding of relative concepts, two skills related to literacy acquisition later in the school-age years. It is also unclear how documented difficulties with executive function, behavioral regulation, and attention contribute to the ongoing struggles with language and academics for children born prematurely $[14,15]$.

\section{Medical and social background}

Ivan is a 4 year, 4-month old male who resides with both parents in New York City, New York, United States. His family qualifies as upper-middle class, allowing them resources for 
advanced medical care and interventions beyond what is provided by local government services. Ivan was born prematurely at 24 weeks' gestation, weighing just 440 grams. Ivan was delivered via emergency cesarean delivery due to mother's diagnosis of Hemolysis Elevated Liver and Low Platelet (HELLP) Syndrome, a form of preeclampsia (gestational high blood pressure and/or hypertension). Ivan was intubated for four months and placed on respiratory support via bubble $\mathrm{C}$ PAP for an additional month in the Neonatal Intensive Care Unit (NICU). Otoacoustic emission testing determined that hearing was within normal limits. Ivan wore a $1 / 4 \mathrm{~L}$ nasal cannula at home until shortly after his first birthday secondary to prolonged respiratory distress syndrome. He continued with ongoing respiratory issues, compromising his overall health, physical development, and vital capacity for speech production. Ivan also had severe eczema, requiring protective covering on his hands until he was 15 months old. These protective hand coverings, coupled with his other medical issues, limited his ability to explore his environment and mouth objects, as a typical child would during this period.

\section{Developmental milestones}

Ivan's complex medical history and activity limitations compounded his slow development. Milestones reported here give both the chronological age and the adjusted age, or chronological age minus the gestational period of prematurity [16]. See Tables 1 and 2 below for gross motor and speechlanguage milestones. Although gross motor milestones were reached within an appropriate to mildly delayed time window, Ivan continued to need support to sustain these skills, often tired quickly performing them, and was noted to be slow or uncoordinated. Although cooing and babbling milestones were reported, Ivan's mother characterized his babbling as infrequent and limited. It was further noted that the first word uttered at 13 months was not repeated and did not signal the onset of vocabulary growth and functional communication. Additionally, Ivan presented with severe oropharyngeal dysphagia and remained bottle fed for his main source of nutrition until approximately 3 years of age.

Table 1. Gross motor milestones

\begin{tabular}{lcc}
\hline Skill & Chronological age & Adjusted age \\
\hline Upright sit & 11 months & 7 months \\
Crawl & 12 months & 8 months \\
Walk & 18 months & 14 months \\
\hline
\end{tabular}

\section{Initial evaluation}

Ivan received speech-language, feeding, physical, and occupational therapy at home through the New York City Early Intervention Program. These services are provided at no cost by the Department of Health to children from birth until approximately 3 years old. Early therapies were focused on increasing nutritional intake to support growth and health status, as well as supporting fine and gross motor skills for activities of daily living. In Ivan's circumstances children in New York City transition from home-based services through the Department of Health to school-based services through the Department of Education. At age 2 years, 6 months $(2 ; 6)$ Ivan was evaluated by a developmental team through the Department of Education to determine eligibility for ongoing speech and language support when he enters preschool.

Administration of the Wechsler Preschool and Primary Scale of Development 4th Edition [17] by the Department of Education evaluation team revealed Working Memory Skills in the 25th percentile (average range) but Visual Spatial Reasoning in the 9th percentile (low average range). Verbal Comprehension and Full Scale Intelligence Quotient were both in the borderline range, in the 3rd and 5th percentiles respectively. Administration of the Preschool Language Scale, 5th Edition [18] found Ivan to be in the 1st percentile, or severe range, for Auditory Comprehension, Expressive Language, and Total Language Score. Noting that Ivan was still nonverbal at age 2;6, evaluators suspected that Ivan may present with Childhood Apraxia of Speech (CAS). His parents were encouraged to seek out speech-language pathologists with specialization in CAS, speech, and language development to further inform the results of the Department of Education evaluation.

\section{Childhood apraxia of speech: uncertain diagnostic boundaries}

CAS is a pediatric motor speech disorder with a core deficit in transcoding, or converting abstract phonological codes to motor speech commands. This core deficit is generally identified by observable speech behaviors, none of which are

Table 2. Speech-language milestones

\begin{tabular}{lcc}
\hline Skill & Chronological age & Adjusted age \\
\hline Coo & 6 months & 2 months \\
Babble & 10 months & 4 months \\
First word & 13 months & 9 months \\
Two word combinations & 21 months & 17 months \\
\hline
\end{tabular}


unique to CAS. Previous surveys of practicing clinicians revealed that over 50 different features are used in determining a CAS diagnosis [19]. Acknowledging difficulties in defining CAS in relation to other pediatric speech sound disorders, the American Speech-Language-Hearing Association (ASHA) [20] provided a consensus statement on the definition of CAS as well as outlined three core perceptual speech features: "1) inconsistent errors on consonants and vowels on repeated productions of syllables and words, 2) lengthened and disrupted coarticulatory transitions between sounds and syllables, and 3) inappropriate prosody, especially in the realization of lexical or phrasal stress" (p. 3). In spite of progress with identifying differentially diagnostic features of CAS [21,22], in the absence of valid and reliable assessment tools, the gold standard remains expert clinical opinion [22,23]. It is assumed that expert clinical opinions are derived from working with many children who have CAS. However, it is estimated that CAS occurs in only one to two children per thousand [24] thus designating it a low incidence disorder. CAS is poorly defined and does not have a validated list of pathognomic features. While population estimates are low, overdiagnosis of CAS is acknowledged in the field $[20,25,26]$. Over-diagnosis, or misdiagnosis, of CAS in-turn shapes clinical experience as the basis of informed clinical opinions. Considering these factors, one source of over-diagnosis of CAS may stem from differing theoretical perspectives held by expert clinicians.

\section{Historical and theoretical perspectives on pediatric speech sound disorders}

Historically, the field of speech-language pathology viewed speech disorders in children as resulting from motoric simplifications or difficulty with motor planning. In the 1970s, the introduction of clinical linguistics began to transform the field of speech-language pathology [27]. The notion of phonological representation began to change our conceptualization of pediatric speech sound disorders, and subsequently language disorders as well $[28,29]$. Upon this innovation, clinicians viewed pediatric speech sound disorder from a clinically dichotomous perspective, in which motor-based speech disorders are seen as separate or distinct from language disorders [30]. Although efforts to unify the constructs under the umbrella term "speech sound disorders" [31] may reflect our current understanding and best practices, this is a recent development. In clinical practice, we continue to see the influence of two distinct traditions: motor-based and language-based. In the motor-based tradition, examination of pediatric speech sound disorders includes a focus on planes of movement of oral musculature; oral-sensory awareness; coordination, strength, and range of motion of articulators. In this view speech errors are seen as motoric simplifications [32,33]. Therefore, expressive communication delays would be at least in part influenced by motoric difficulties. In the linguistic tradition there is a focus on intentional communication, and the cognitive-linguistic precursors to expressive speech and language (e.g. play, symbolic gestures) [34]. Speech sound errors would be considered deficits in linguistic representation, particularly in the phonological system. Given the historical shifts in the field's views on speech disorders, individual clinicians' conceptualizations of pediatric speech sound disorders may be a product of the era in which they were trained or the philosophies imparted to them by their training programs $[35,36]$. Although there is room to allow both theoretical perspectives, it should be noted that some hold-overs of the motor tradition, such as non-speech oral motor exercises are still used by many clinicians despite a limited evidence base $[35,37,38]$.

For a low-incidence disorder with uncertain diagnostic boundaries and limited validated assessment protocols, clinicians charged with differentially diagnosing cases of CAS will likely be reliant on their own theoretical perspectives. Hence, clinicians adopting a motor learning philosophy will emphasize the behavioral aspects of motor learning and execution motor plans for speech production. Aligned with this view, theoretically the acquisition of motor plans requires good proprioception to learn what speech production movements sound and feel like [39,40]. Clinicians from a more linguistic tradition will often approach assessment utilizing languagebased strategies, consistent with psycholinguistic speech processing models tied to neurobiological underpinnings of language disorders. With this approach, expressive output would be characterized by the relationship between the underlying representations of lexical and phonological forms preceding the execution of a motor plan [41]. Language-based clinicians will focus on cognitive precursors to communication and communicative intent in the context of symbolic play schemas [34].

What follows are summaries of evaluations and treatment plans for Ivan at chronological age 2;7 by two clinicians with different training and perspectives, both of whom have specialization in the diagnosis of CAS. The period of treatment reported here for comparison between the two clinicians is 3 months, while Ivan was aged 2;7-2;10. Clinician 1 is a CAS expert with specializations in oral-motor and feeding difficul- 
ties. Clinician 2 is also a CAS expert, but with a background in linguistics. Ivan's complex development was interpreted differently by clinicians coming from distinct traditions, and resulted in distinct intervention approaches. Aligned with the aforementioned, Clinician 1 with a motor-based perspective focused on oral motor movements and speech sound production and intelligibility, while Clinician 2, a language-based clinician, focused on communicative intent, functional communication and symbolic play skills. Following a summary of their approaches to assessment and intervention, this report then summarizes and integrates the observations across the two professionals, while honoring their perspectives.

\section{Assessment and treatment plans \\ Clinician 1: motor based assessment}

Clinician 1 performed a motor-based assessment focusing on sensory and motor processing and speech sound production. An oral peripheral examination revealed reduced tone in cheeks, lips, and tongue. Ivan presented with tactile defensiveness in the peri-oral area and hyper-sensitivity in the intra-oral system but he tolerated firm touch on the posterior teeth. He accepted stimulation in the buccal cavity with a trimmed toothette. Reduced strength was documented in the masseter muscles, which was noted as contributing to jaw instability. Reduced lip rounding and reduced strength and range of motion of tongue were also noted. Clinician 1 probed use of tactile-kinesthetic feedback cueing system (Prompts for Restructuring Oral Motor Targets; PROMPT [42]) which resulted in imitation of vowel and bilabial sounds. Poor motor planning for speech sounds and poor oral placement skills were noted. It was also noted that Ivan could not blow bubbles. Based on the oral motor assessment, Clinician 1 concluded that Ivan presented with CAS, citing difficulty achieving and maintaining articulatory placement, presence of vowel distortions, limited consonant and vowel repertoire, and use of simple syllable shapes. It was also noted that Ivan presented with concomitant dysarthria, with oral-muscular weakness.

\section{Motor based treatment plan}

Based on evaluation results, Clinician 1 developed a motor based treatment plan for Ivan at ages 2;7-2;10. The motorbased treatment included the targets outlined below to in- crease speech production and intelligibility. Clinician 1 also worked on feeding goals to increase oral intake and improve cup drinking. Feeding goals and treatment plan are not reported here. Speech goals included: Production of vowel consonant (VC) syllables with visual and verbal feedback; Production of vowel /o/ to request "open" during play; Production of consonant vowel (CV) syllable /wa/ for "one" and /mu/ "moo" for cow during play. Speech goals were to be augmented with intra-oral stimulation and oral-motor exercises, utilizing a variety of oral-motor tools (vibrators, chewytubes, toothettes $)^{1)}$. Activities included saying /wa/, making kissing motions, chewing on tubes, and blowing. Over the 3-month period reported here, Ivan was seen by Clinician 1 twice weekly for 30 minutes. Progress reported by Clinician 1 under this treatment program include increased production of CV and VC syllables, increased tolerance of peri-oral and intra-oral sensation, and increased verbal imitation of speech.

\section{Clinician 2: language based assessment}

Clinician 2 performed a play-based assessment focusing on symbolic play skills and communicative intent. As the child was less than 3 years old, and his speech sound errors were still part of typical phonological development, this assessment did not focus on speech intelligibility and articulation. During play-based tasks, Ivan demonstrated some eye contact but limited communicative intent, evidenced by lack of gesture or sign. Ivan did not respond to hand-over-hand techniques to produce sign or gesture. Ivan also presented with limited joint attention; he did not participate in play routines (e.g. peek-aboo games) and also did not persist to request a desired toy that was withheld. Observed play skills were limited to rolling cars back and forth, and efforts to engage Ivan in reciprocal and symbolic play were ignored or rejected. Word production was limited. Ivan did not imitate words. It was noted that with the few words elicited that Ivan had difficulty coordinating his breath with voice, resulting in low volume. Oral motor strength and range of motion were not directly assessed, but low tone was observed in oral-motor movements. Clinician 2 proposed a diagnosis of expressive language delay with concomitant mild dysarthria, to be further evaluated with diagnostic therapy. Clinician 2 cited difficulty with social-pragmatic aspects of language and limited play skills.

\footnotetext{
1) The author of this manuscript does not condone use of oral motor exercises for remediation of speech sound production. See Lass \& Pannabacker (2008) for a review $[37,42,41]$.
} 


\section{Language based treatment plan}

Based on evaluation results, Clinician 2 developed a language and play-based treatment plan for Ivan at ages 2;7-2;10. Targets for this treatment plan are outlined below, with the goal to increase play skills and communicative behavior such as joint attention and requesting. Initial goals included: Imitation of manual signs in context of request (more/open); Imitation of 3-step sequences of actions in the context of play (open box, stack blocks from inside, knock down block structure); Coordination of breath with voice during play (singing, choochoo and vroom vroom sounds with trains and cars). Over the 3 month period reported here, Ivan was seen by Clinician 2 once weekly for 60 minutes. Ivan made slow progress with initial goals but early treatment outcomes included: Anticipation of actions in 3-step routines; Improved vocal loudness and coordination with breath; Utilization of manual signs with a model; Pairing of single words with manual signs, given exaggerated models.

Upon turning 3 years old Ivan began attending preschool three mornings per week and had his first consistent exposure to same-age peers. During this time, Ivan added over 20 words to his expressive vocabulary. He quickly progressed to using single word utterances independently and began combining two words given a delayed model. Ivan's rapid vocabulary spurt when he was given increased opportunities to socialize with peers supports the view that there was a social-pragmatic aspect to his delayed onset of expressive language.

\section{Update on speech and language development}

Almost 1 year following the initial evaluations, at chronological age 3;10, Ivan received an additional follow-up evaluation by the NYC Department of Education. As his communication skills had improved, his test scores in other domains were higher. Cognitive testing indicated that Ivan was in the 93rd percentile, above average for intellectual functioning. Speechlanguage evaluation indicated that Ivan continued with poor oral motor control and coordination, contributing to speech and feeding difficulties. Ivan presented with mostly age-appropriate speech sound errors but intelligibility was further compromised by low volume. Speech errors at this time were consistent with developmental phonological processes in English (e.g. stopping, fronting, cluster reductions). Spontaneous expressive language was characterized by four-word utterances to comment and request. He was observed to use some age-appropriate English morphological markers (present progressive, plural, regular past tense) but did not use auxiliary verb forms. Receptive language skills included the ability to follow two-step commands and respond to questions with short utterances. His ability to make inferences was emerging. Ivan's play skills had improved and included some symbolic play when guided by an adult. He continued to struggle using language socially with peers and participating in play schemas requiring communicative interactions. With respect to the literature on speech development in preterm and very low birthweight children reviewed here, Ivan fit the profile of an early severe gap in speech sound development compared to peers that narrows over time [9-11]. Similarly, although his expressive language skills had advanced, he was still moderately-severely delayed compared to peers and at-risk for academic difficulties, as noted by several sources $[6,13]$.

\section{DISCUSSION}

Ivan is a young child who was born extremely preterm at a very low birthweight. Given his rare circumstances and the evolving nature medical management of children born before the period of viability, there is limited information in the literature to guide clinicians' assessment and treatment of his speech and language. He presented with a mixed profile of oral-motor, speech, language, and social deficits. Two distinct diagnoses and treatment plans were derived from separate evaluations: Childhood Apraxia of Speech vs. Expressive Language Disorder. In the context of a CAS diagnosis, in the absence of a statement regarding language development, it is presumed that the root of his limited verbal output and social interactions lay with speech motor planning deficits. With the Expressive Language Disorder diagnosis, it is presumed that the root of limited verbal output and social interactions lay with deficits in the comprehension, use, and organization of the language system. The observations of the two dichotomous perspectives are not mutually exclusive. The limited repertoire of phonemes and syllable shapes noted by Clinician 1 is not contradictory to the limited verbal output noted by Clinician 2 . However, the polarized foundations of these observations resulted in distinct treatment plans. Clinician 1 focused narrowly on articulatory placement and building communication through consonant-vowel (CV) syllables, while Clinician 2 focused more broadly on communicative intent by building symbolic representation via manual sign and play schemas.

The relationships between speech motor and linguistic development are still poorly understood [43]. In typically devel- 
oping children linguistic units at the syllable, phrase, and sentence-level drive speech movement output, with a protracted developmental trajectory extending into early adolescence [44]. In children with communication disorders, both higherorder linguistic deficits and lower-level motor planning deficits may disrupt the integration of motor speech and linguistic substrates, contributing to profiles of either expressive language or motor speech disorders respectively [21]. Although a stark clinical dichotomy was present in this case, the child was treated by both clinicians and made substantial gains in treatment. Both clinicians assessed and treated Ivan utilizing the evidence base inherent to their own views on communication disorders and training. While working in their respective traditions, both clinicians' plans overlapped by initially targeting joint attention and requesting. Although the later presentation of expressive language impairment with social communication deficits may have been better addressed by a language-based approach, we cannot isolate the gains made from this approach from the work done simultaneously on motor speech production. For example, it is possible that the gains made in speech imitation under the motor-based approach facilitated the interactions necessary for the gains in productive vocabulary with the language-based approach [45]. Likewise, it is possible that focus on imitating actions during play schemas precipitated gains in didactic communication to facilitate speech imitation [46]. However, as Ivan matured the preliminary signs of CAS resolved. Thus, the evidence-based treatment for CAS, including intensive treatment with high repetitions of target speech sounds [23,47,48], likely would not have facilitated improvement in expressive vocabulary, morphosyntax, or social communication.

In this particular case, it is possible that the severity and nature of the child's impairment necessitated approaches that spanned both speech-motor and linguistic elements before age three. However, it is rare for a child to be treated by separate clinicians simultaneously. We do not know the outcomes for premature children who are treated by only one clinician with either a wholly motor or linguistic approach. Furthermore, caution should be applied when utilizing purely motorbased approaches to ensure methods are evidence-based and address overall linguistic development. This case report urges consideration of how divergent perspectives on speech and language development exacerbate the impact of poorly defined diagnostic boundaries. The discrepancy in diagnoses and approaches not only calls for more research regarding the interaction of motor and linguistic behavior during develop- ment, descriptive studies of premature children, and diagnostic boundaries of CAS, but also further introspection of how our clinical opinions are formed through our training and practice.

\section{REFERENCES}

1. Anderson JG, Baer RJ, Partridge JC, Kuppermann M, Franck LS, Rand L, et al. Survival and major morbidity of extremely preterm infants: a population-based study. Pediatrics. 2016:e20154434.

2. Hoekstra RE, Ferrara TB, Couser RJ, Payne NR, Connett JE. Survival and long-term neurodevelopmental outcome of extremely premature infants born at 23-26 weeks' gestational age at a tertiary center. Pediatrics. 2004;113(1):e1-e6.

3. Poehlmann J, Schwichtenberg A, Bolt DM, Hane A, Burnson C, Winters J. Infant physiological regulation and maternal risks as predictors of dyadic interaction trajectories in families with a preterm infant. Developmental Psychology. 2011;47(1):91.

4. Jansson-Verkasalo E, Ruusuvirta T, Huotilainen M, Alku P, Kushnerenko E, Suominen K, et al. Atypical perceptual narrowing in prematurely born infants is associated with compromised language acquisition at 2 years of age. BMC Neuroscience. 2010;11(1): 88.

5. Reissland N, Stephenson T. Turn-taking in early vocal interaction: a comparison of premature and term infants' vocal interaction with their mothers. Child: Care, Health and Development. 1999; 25(6):447-456.

6. Foster-Cohen S, Edgin JO, Champion PR, Woodward LJ. Early delayed language development in very preterm infants: evidence from the MacArthur-Bates CDI. Journal of Child Language. 2007; 34(3):655-675.

7. Brown BB, Bendersky M, Chapman T. The early utterances of preterm infants. British Journal of Disorders of Communication. 1986;21(3):307-319.

8. Wolke D, Meyer R. Cognitive status, language attainment, and prereading skills of 6-year-old very preterm children and their peers: the Bavarian Longitudinal Study. Developmental Medicine and Child Neurology. 1999;41(2):94-109.

9. Rickards A, Ford GW, Kitchen WH, Doyle L, Lissenden J, Keith C. Extremely-low-birthweight infants: neurological, psychological, growth and health status beyond five years of age. The Medical Journal of Australia. 1987;147(10):476-481.

10. Klein NK, Hack M, Breslau N. Children who were very low birth weight: development and academic achievement at nine years of age. Journal of Developmental \& Behavioral Pediatrics. 1989; 10(1):32-37.

11. Aram DM, Hack M, Hawkins S, Weissman BM, Borawski-Clark E. Very-low-birthweight children and speech and language development. Journal of Speech, Language, and Hearing Research. 1991; 34(5):1169-1179.

12. Vohr BR, Wright LL, Dusick AM, Mele L, Verter J, Steichen JJ, et al. Neurodevelopmental and functional outcomes of extremely low 
birth weight infants in the National Institute of Child Health and Human Development Neonatal Research Network, 1993-1994. Pediatrics. 2000;105(6):1216-1226.

13. Luoma L. Speech and language development of children born at 32 weeks' gestation: a 5-year prospective follow-up study. Developmental Medicine \& Child Neurology. 1998;40(6):380-387.

14. Anderson P, Doyle LW, Group VICS. Neurobehavioral outcomes of school-age children born extremely low birth weight or very preterm in the 1990s. Jama. 2003;289(24):3264-3272.

15. Aarnoudse-Moens CSH, Weisglas-Kuperus N, van Goudoever JB, Oosterlaan J. Meta-analysis of neurobehavioral outcomes in very preterm and/or very low birth weight children. Pediatrics. 2009; 124(2):717-728.

16. Palisano RJ. Use of chronological and adjusted ages to compare motor development of healthy preterm and fullterm infants. Developmental Medicine \& Child Neurology. 1986;28(2):180-187.

17. Wechsler D. Wechsler Preschool and Primary Scale of Intelligence - Revised. 1989.

18. Zimmerman IL, Steiner VG, Pond E. Preschool Language ScalesFifth Edition (PLS-5). San Antonio, TX: Pearson. 2011.

19. Forrest K. Diagnostic criteria of developmental apraxia of speech used by clinical speech-language pathologists. American Journal of Speech-Language Pathology. 2003;12(3):376-380.

20. American Speech-Language-Hearing Association Childhood Apraxia of Speech: [Technical Report]. Rockville Pike, MD: ASHA; 2007.

21. Iuzzini-Seigel J, Hogan TP, Green JR. Speech inconsistency in children with childhood apraxia of speech, language impairment, and speech delay: sepends on the stimuli. Journal of Speech, Language, and Hearing Research. 2017;60(5):1194-1210.

22. Murray E, McCabe P, Heard R, Ballard KJ. Differential diagnosis of children with suspected childhood apraxia of speech. J Speech Lang Hear Res. 2015;58(1):43-60.

23. Maas E, Butalla CE, Farinella KA. Feedback Frequency in Treatment for Childhood Apraxia of Speech. American Journal of Speech-Language Pathology. 2012;21(3):239-257.

24. Shriberg LD, Aram DM, Kwiatkowski J. Developmental apraxia of speech: I. descriptive and theoretical perspectives. Journal of Speech, Language, and Hearing Research. 1997;40(2):273-285.

25. Davis BL, Jakielski KJ, Marquardt TP. Developmental apraxia of speech: determiners of differential diagnosis. Clinical Linguistics \& Phonetics. 1998;12(1):25-45.

26. Shriberg LD, Potter NL, Strand EA. Prevalence and phenotype of childhood apraxia of speech in youth with galactosemia. Journal of Speech Language and Hearing Research. 2011;54(2):487-519.

27. Ingram D. Phonological disability in children: Elsevier Publishing Company; 1977.

28. Fey ME. Articulation and phonology: inextricable constructs in speech pathology. Language, Speech, and Hearing Services in Schools. 1992;23(3):225-232.

29. Kamhi AG. The need for a broad-based model of phonological disorders. Language, Speech, and Hearing Services in Schools. 1992;23(3):261-268.
30. Martin AD. Some objections to the term apraxia of speech. Journal of Speech and Hearing Disorders. 1974;39(1):53-64.

31. American Speech Language Hearing Association (ASHA). Speech sound disorders: articulation and phonology. (Practice Portal) n.d.

32. Bahr D, Rosenfeld-Johnson S. Treatment of children with speech oral placement disorders (OPDs): a paradigm emerges. Communication Disorders Quarterly. 2010;31(3):131-138.

33. Strand EA, editor Treatment of motor speech disorders in children. Seminars in Speech and Language; 1995: @ 1995 by Thieme Medical Publishers, Inc.

34. Westby CE. Assessment of cognitive and language abilities through play. Language, Speech, and Hearing Services in Schools. 1980;11 (3):154-168.

35. Watson MM, Lof GL. A survey of university professors teaching speech sound disorders: nonspeech oral motor exercises and other topics. Language, Speech, and Hearing Services in Schools. 2009;40(3):256-270.

36. Zipoli RP, Kennedy M. Evidence-based practice among speechlanguage pathologists: attitudes, utilization, and barriers. American Journal of Speech-Language Pathology. 2005;14(3):208-220.

37. Lass NJ, Pannbacker M. The application of evidence-based practice to nonspeech oral motor treatments. Language, Speech, and Hearing Services in Schools. 2008;39(3):408-421.

38. Lof GL, Watson MM. A nationwide survey of nonspeech oral motor exercise use: implications for evidence-based practice. Language, Speech, and Hearing Services in Schools. 2008;39(3):392407.

39. Schmidt RA. A schema theory of discrete motor skill learning. Psychological review. 1975;82(4):225.

40. Maas E, Robin DA, Hula SNA, Freedman SE, Wulf G, Ballard KJ, et al. Principles of motor learning in treatment of motor speech disorders. American Journal of Speech-Language Pathology. 2008; 17(3):277-298.

41. Stackhouse J, Wells B. Children's speech and literacy difficulties: a psycholinguistic framework: Wiley; 1997.

42. Hayden D. The PROMPT model: use and application for children with mixed phonological-motor impairment. Advances in Speech Language Pathology. 2006;8(3):265-281.

43. Iverson JM. Developing language in a developing body: the relationship between motor development and language development. Journal of Child Language. 2010;37(2):229-261.

44. Smith A. Speech motor development: integrating muscles, movements, and linguistic units. Journal of Communication Disorders. 2006;39(5):331-349.

45. Kuhl PK, Meltzoff AN. Infant vocalizations in response to speech: vocal imitation and developmental change. The Journal of the Acoustical Society of America. 1996;100(4):2425-2438.

46. Normand M. A developmental exploration of language used to accompany symbolic play in young, normal children (2-4 years old). Child: Care, Health and Development. 1986;12(2):121-134.

47. Maas E. Conditions of practice and feedback in treatment for apraxia of speech. Perspectives on Neurophysiology and Neuro- 
Randazzo M Divergent perspectives extremely preterm birth

genic Speech and Language Disorders. 2010;20(3):81-87.

48. Edeal DM, Gildersleeve-Neumann CE. The Importance of production frequency in therapy for childhood apraxia of speech.
American Journal of Speech-Language Pathology. 2011;20(2):85-

110. 